\title{
EDITORIAL
}

\section{Highlights in agri-product quality and safety}

\author{
Guanghong ZHOU (凶) \\ College of Food Science and Technology, Nanjing Agricultural University, Nanjing 210095, China
}

Agri-product quality and safety are hot topics at the forefront of discussion and concern among governments, researchers and the public. Potential hazards in agri-product include pathogens, antibiotics, pesticides, industrial pollutants, allergens as well as chemicals produced during agri-product processing. In terms of agri-product quality, nutritional value has been widely considered in the past, and until recently this has been the aspect of agri-product quality that has been considered of most importance. This special issue of Frontiers of Agricultural Science and Engineering (FASE) features eleven articles on food of plant and animal origin encompassing a wide range of current research topics on food safety regulation, the mechanisms, prevention and control of food hazards, and nutritional evaluation of food components.

Concerning agri-product safety, Ziping Wu reviews different technical and socioeconomic factors that affect antimicrobial use at the farm level. Wu emphasizes that antimicrobial use in food animals is associated with animal health improvement, economic costs and benefits relevant to animal diseases, and potential technological alternatives. Additionally, antimicrobial use has strong regional and species differences. James N. Seiber provides a comprehensive review on the current status and future prospects of biopesticides, including how their use may increase the quality and safety of the food supply. In two other reviews, the authors discuss the sources, toxicology and elimination of methylglyoxal and other toxic substances of the Maillard reaction in food systems, with a focus on the inhibitory effects of polyphenols and other natural antioxidants.

In addition to chemical contamination, microbiological contamination, allergens and the potential of transgenic animals continue to be important areas of inquiry. In their study, Li, Offengenden, Gänzle and Wu observe that Aspergillus oryzae treatment lowers IgE-binding ability of egg white proteins by cleaving a fragment $(\sim 1700 \mathrm{Da})$ at the N-terminus. Liu, Shang, Yang, Zhang, Lu and Li report the construction of a milk expression vector to successfully generate two transgenic mouse lines that express recombinant human lysozyme (rhLZ) that exhibits the same antibacterial activity as native hLZ in milk. Chen, Zhu, Qin, Qiu and Zhao present the taxonomy, pathogenesis, resistance, detection and control of Cronobacter spp.

Agri-product quality and safety control is not only a technological issue but is also a significant social concern. Joseph J. Jen discusses the challenges facing the food industry, academia and government in China and presents potential solutions to the global challenges for food safety.

With respect to nutrition, Arvik, Kim, Seiber and Yokoyama review the different pleiotropic effects of grape seed procyanidins on the physiology of animal models of obesity. They found that procyanidins are not degraded or metabolized until reaching the lower gut where they can be metabolized by gut bacteria and, in turn, alter the composition of gut bacteria and thus decrease obesity. Nsor-Atindana, Chen, Wei, Noe, Li and Zhong present different in vitro digestion techniques with particular interest in starch digestion in the presence of dietary fiber during each of the three phases of digestion: oral, gastric and intestinal. Li M, Li C, Song, Xu and Zhou present a proteome study on biochemical and histological changes in rat jejunum in response to different dietary proteins. They found that intake of chicken protein can regulate oligopeptide transport mainly by upregulating PEPT1 protein expression and reducing dipeptidyl-peptidase activity related to biological oxidation, but it also can reduce oligopeptide

Received June 1, 2018; accepted June 15, 2018

Correspondence: ghzhou@njau.edu.cn

(C) The Author(s) 2018. Published by Higher Education Press. This is an open access article under the CC BY license http://creativecommons.org/licenses/by/4.0 
absorption capacity by regulating the Hippo signaling pathway.

Finally, as the Guest Editor, I would like to thank the authors and reviewers who have given their time, energy and expertise to this special issue, as well as the FASE editorial team for their valuable input and unfailing support.

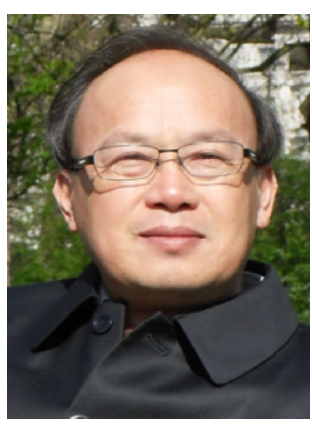

Dr. Guanghong Zhou, a distinguished professor and president of Nanjing Agricultural University, is a prominent international scholar in food science and technology. He is widely recognized for his original work to elucidate the biochemical mechanisms behind the texture quality of muscle foods and flavor formation in traditional Chinese meat products. He has successfully conducted several national research programs regarding to quality and safety control on chilled fresh meat and Chinese traditional food products. His research has made exemplary contributions to China's meat industry. In addition, he led the drafting of three UNECE international meat standards, as well as dozens of national or industrial food standards in China. He was appointed as ISO chairperson for meat, poultry, fish, eggs and their products in 2017, and elected IFT Fellow and IAFoST Fellow in 2018. 